\title{
Optical properties of metallo-dielectric microspheres in opal structures
}

\author{
Y Jiang, C Whitehouse, Jensen Li, Wing Yim Tam, \\ C T Chan and Ping Sheng \\ Department of Physics, Hong Kong University of Science and Technology, Clear Water Bay, \\ Kowloon, Hong Kong, People's Republic of China
}

Received 13 March 2003, in final form 8 July 2003

Published 15 August 2003

Online at stacks.iop.org/JPhysCM/15/5871

\begin{abstract}
We report the fabrication of opal structure using metallo-dielectric silica microspheres. Mono-dispersed silica microspheres were coated with silver using an electrode-less wet-plating technique. Thin slabs of opal were obtained by assembling the silver-coated microspheres between two glass plates using a forced-packing method. The optical properties of the resulting opal structure were studied in the infrared range. Good agreement is obtained with the predictions of a multiple scattering approach, provided that the silver layer is modelled as a silver composite.
\end{abstract}

\section{Introduction}

Recent developments in photonic band gap (PBG) materials have attracted much interest in basic and applied research. These materials have frequency ranges in which electromagnetic waves cannot propagate, in analogy to the electronic forbidden gaps in semiconductors [1]. PBG materials can be realized in two component dielectric media with a periodic variation in the refractive index. Two promising routes that have been used to make PBG crystals in the IR/optical frequency ranges are: (i) microfabrication [2] and (ii) inverse-opal and related techniques [3]. These two methods are very different in principle and in practice, but both seek to create some predefined artificial periodic array of high dielectric materials. Recently, a new approach focusing on the building blocks rather than the periodic order, has been reported in which metallo-dielectric spheres were used $[4,5]$. Complete band gaps were realized in various periodic structures in the microwave regime, in good agreement with the theoretical predictions [5]. Furthermore, it was shown that by reducing the size of the coated spheres, band gaps in the visible regime may also be realized by using silver as the metallic component. Here we report the fabrication of periodic structures using metal-coated silica microspheres. The optical properties of these structures were studied in the infrared regime. In what follows, details of the fabrication and optical measurements are presented in the experimental section 2. Theoretical modelling of the system is given in section 3, followed by a discussion. 


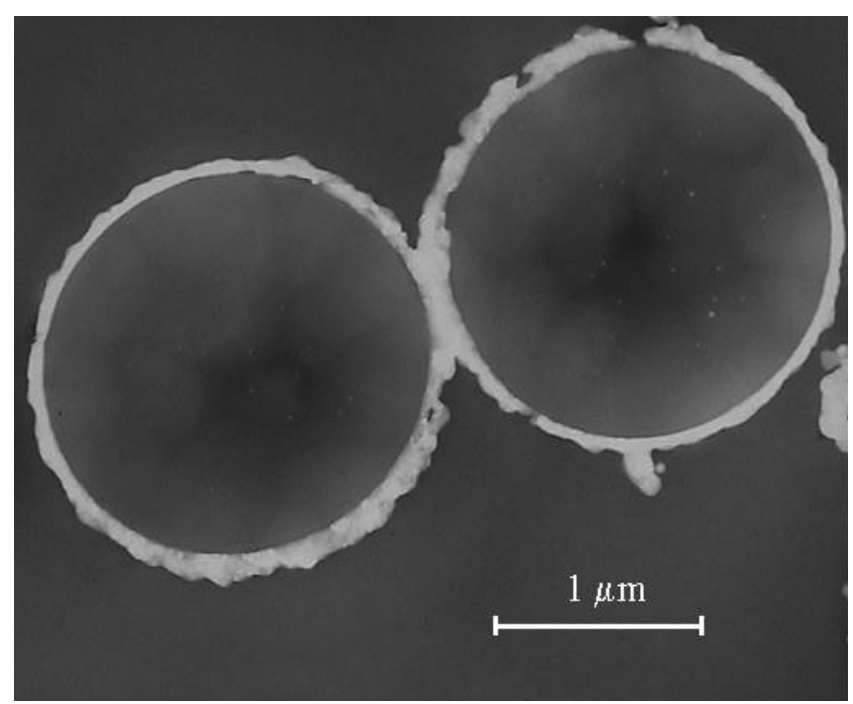

Figure 1. TEM image of cross-sections of $2 \mu \mathrm{m}$ silver-coated silica microspheres. (The image was taken using a Philips CM20 TEM with a sample prepared by ion milling coated particles set in epoxy resin.)

\section{Experimental details}

To start, $2.0 \mu \mathrm{m}$ diameter silica $\left(\mathrm{SiO}_{2}\right)$ spheres (from Sekisui Chemical Co., Ltd) with a size dispersion of better than $3 \%$ were used as the core particles. These silica microspheres were coated with an outer layer of silver using an electrode-less wet-plating method [6]. Silver was chosen because it is the least dissipative metal in the working frequency range. The silica particles were first cleaned in a $5 \% \mathrm{H}_{2} \mathrm{SO}_{4}$ and $0.05 \% \mathrm{HF}$ solution for $5 \mathrm{~min}$ and then treated with a $0.06 \mathrm{~g} \mathrm{l}^{-1} \mathrm{SnCl}_{2}$ solution for $15 \mathrm{~min}$ prior to plating. The size of the silica spheres was reduced to about $1.8 \mu \mathrm{m}$ after the cleaning process. The plating reaction took place at ambient temperature under sonic and manual agitation to ensure the particles were coated uniformly and individually to a final size of $2 \mu \mathrm{m}$ in diameter. The silver plating bath contained components in the molar ratio $\mathrm{Ag} / \mathrm{Na} / \mathrm{RA} / \mathrm{Si}=1 / 6.9-41.4 / 0.42-2.5 / 0.02-0.71 .{ }^{1}$ The coated particles were separated out by fractional filtering after being rinsed many times with distilled water. The resulting particles, of about $5 \%$ dispersity, showed silver coatings with thicknesses in the range 70-160 $\mathrm{nm}$ as measured from the TEM image shown in figure 1, and calculated from EDX measurements. The Auger electron parameter values confirmed the presence of silver in metallic form [7]. As shown in figure 1, the silver coating has a surface roughness in tens of nanometres. However, the particles display high reflectivity under an optical microscope.

Sedimentation is commonly used to assemble mono-dispersed mesoscale particles to form periodic structures. However, this method usually takes a long time and there is little control over the resulting morphology of the packed assembly. Recently, a force-packing method was successfully employed in fabricating large-scale (about $1 \mathrm{~cm}^{2}$ ) multilayer assemblies, with a well-defined number of layers [8]. Figure 2 shows the schematic of the forcing-packing chamber used in this work to pack the silver-coated microspheres. The chamber consisted of a bottom silicon wafer and a top glass plate. A rectangular frame of photoresist deposited onto the silicon wafer formed the side walls of the cell. At one side of the frame, shallow

1 We used $\mathrm{AgNO}_{3}$ for the source of silver, $\mathrm{NaOH}$ for the alkaline medium and glucose for the reducing agent (RA). 


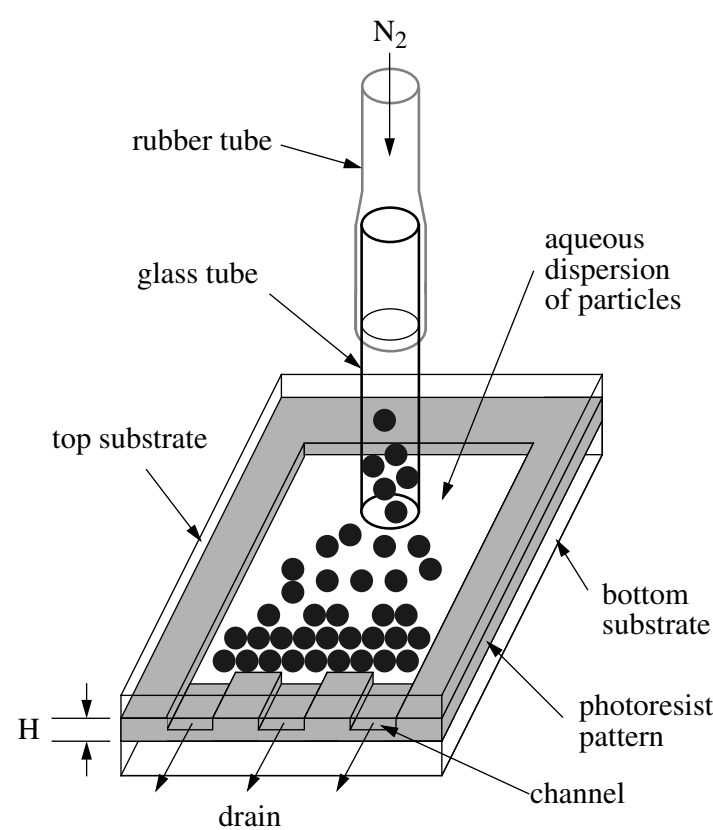

Figure 2. Schematic setup for forced packing of microspheres.

trenches were etched out of the photoresist. These trenches allowed fluid to pass but restrained the packing particles once the top glass plate was installed. The top glass plate had a small hole with a glass tube attached, which was connected to a nitrogen gas supply at a controlled pressure through rubber tubing. The glass tube provided the inlet for the particle suspension. The top glass and the bottom silicon plates were held together with binder clips to form a thin chamber such that it was completely sealed except at the side with the trenches. The whole assembly was submerged in a sonic water bath. The silver-coated microspheres were first dispersed in distilled water to form a suspension with a low particle concentration. The suspension was then introduced into the chamber by injection through the rubber tubing with a syringe whilst nitrogen flowed slowly through the chamber. The particles began to assemble starting at the side with the trenches. Several injections might be needed to obtain a large working area of ordered structure. It is important to adjust the sonic power and to maintain its level during the packing period to obtain well-packed structures. After the sample had reached a workable size, the filling process was stopped and the whole assembly was allowed to dry in open air. The final sample was obtained by removing the top glass plate leaving behind the particle assembly supported by the silicon plate. To avoid disrupting the packed assembly the top glass plate was coated with a non-stick material [8]. Samples with domain sizes of the order of $30 \times 30$ particles were easily obtained as shown in figure 3 , in which we show an optical microscope image of a three-layer FCC structure in the [111] direction consisting of the silver-coated microspheres. Occasionally, domains with the [100] face parallel to the substrate could also be obtained. Samples of uncoated silica microspheres were also obtained using the same method for comparison purposes.

The reflectance and transmittance were measured at normal incidence using a Fourier transform IR spectrometer (FTIR, BIO-RAD FTS 6000) coupled with an IR microscope (UMA500). The microscope can focus down to an area of $25 \times 25 \mu \mathrm{m}^{2}$, small enough to obtain 


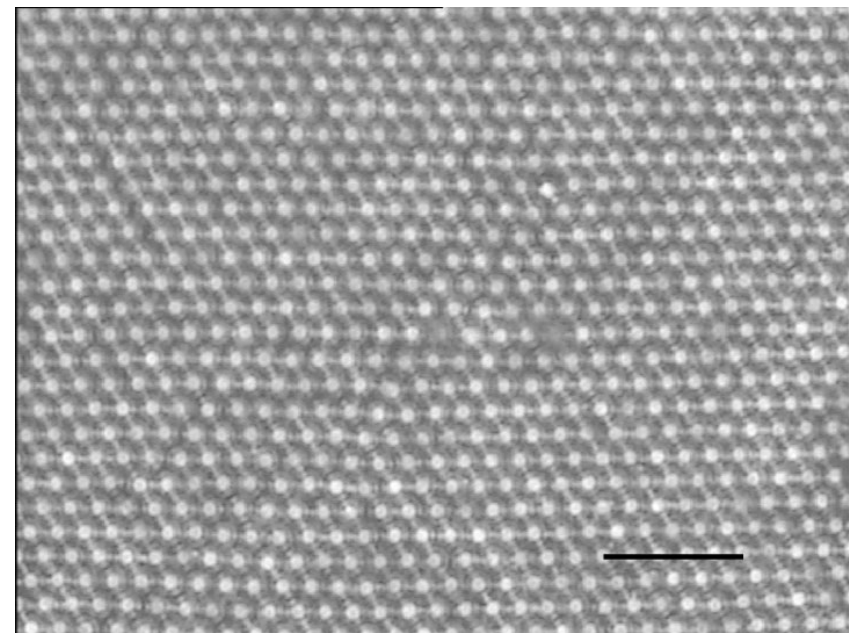

Figure 3. Optical image of $2 \mu \mathrm{m}$ silver coated silica microspheres packed in FCC structure along the [111] direction. The scale bar is $10 \mu \mathrm{m}$.

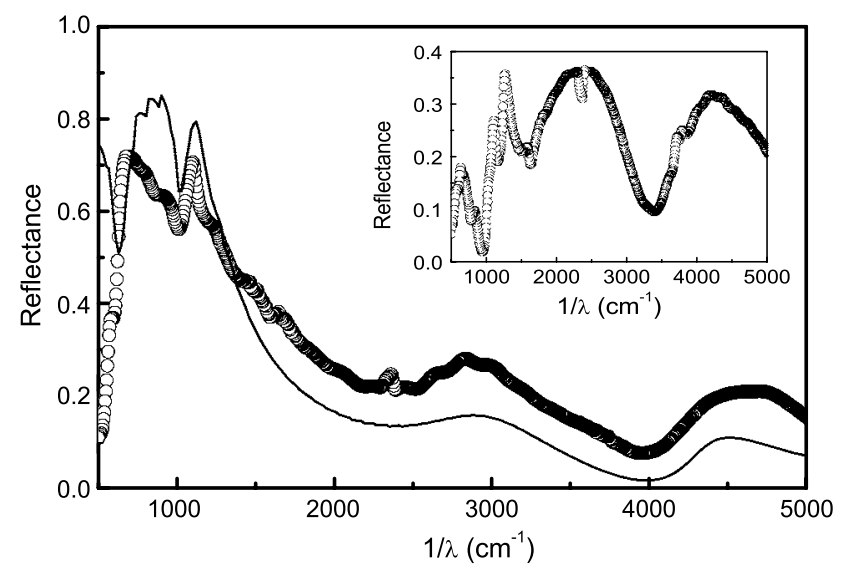

Figure 4. Reflectance of $2 \mu \mathrm{m}$ silver-coated silica microspheres for an FCC opal three layers thick along the [111] direction for the experiment (solid dots) and multiple-scattering calculation (solid curve). The coating layer is modelled as an Ag-dielectric symmetric composite (see text for details). The inset shows the reflectance of an FCC opal using uncoated silica microspheres. The feature at $2300 \mathrm{~cm}^{-1}$ is due to $\mathrm{CO}_{2}$.

data from a single domain. A standard ceramic MID-IR source and a liquid-nitrogen-cooled photodetector were used to cover a wavelength range of 2-20 $\mu \mathrm{m}$. A gold mirror was used as a reflectance reference while a silicon wafer provided a transmittance reference. Figure 4 shows the normalized reflectance of packed microspheres in the [111] direction. Characteristic features, namely broad peaks and dips, were observed. However, due to the high reflectance around $1000 \mathrm{~cm}^{-1}$ and large absorption at higher wavenumbers, the transmittance is too small to exhibit any clear feature. For comparison, the inset of figure 4 shows the FTIR reflectance of opal made with the uncoated silica microspheres. There is a characteristic $\mathrm{SiO}_{2}$ absorption around $1000 \mathrm{~cm}^{-1}$. The signature of this $\mathrm{SiO}_{2}$ absorption can also be found in the silver-coated microspheres, but becomes much weaker because of the shielding effect of the Ag layer. 


\section{Calculation}

In order to interpret the experimentally measured spectrum, we use a multiple-scattering formalism that is best suited for a collection of spherical objects. Our model system was composed of closely packed metal-coated microspheres in the FCC structure. Each sphere has an $\mathrm{SiO}_{2}$ core, $0.9 \mu \mathrm{m}$ in radius, with an average silver coating of thickness $0.1 \mu \mathrm{m}$. The mathematical details of the multiple-scattering formulation can be found elsewhere [9]. It has been applied successfully to the calculation of optical properties for systems consisting of metal or metal-coated spheres [5]. The multiple-scattering approach can be formulated as a band structure technique [9] that gives the dispersion of light inside a bulk sample. It can also be formulated to calculate transmission and reflection coefficients [10] when light is incident on a particular orientation of a slab. As noted below, the band structure results ignore absorption (but include frequency dispersion), while the transmission/reflection calculation can take dispersion and absorption fully into account. After extensive calculations, we found that good agreement between theory and experiment can be obtained if we model the metal not as an ideal layer of pure silver, but as a composite of silver and a dielectric. This finding is consistent with the electron microscope pictures of the silver-coated spheres showing a spongy appearance. We thus model the silver layer as a spongy structure containing silver and air. Since the mircostructure of the Ag-dielectric layer is significantly smaller than the wavelength of the incident light, the coating layer can be modelled as an effective medium with effective dielectric constants given by the Bruggeman formula [11] ${ }^{2}$. Both the dielectric constants of the glass core and silver (as part of the composite) are taken from measured values as reported in handbooks of optical constants [12]. We find the best agreement between theory and experiment when the ratio of silver to air is 0.35 .

The theoretical results are plotted for comparison with the experimental results in figure 4 . The calculated reflection coefficients for three layers of the closely packed particles (stacked along the [111] direction of an FCC lattice) show salient features that agree well with the experimental result. It is noted that the results are rather insensitive to the dielectric constant of the dielectric constituent in the Ag coating layer. The results in figure 4 simply take $n=1$. When the 'air' in the spongy layer of silver is replaced by a dielectric with $n=1.45$, the results are qualitatively the same, as shown in figure 5. However, the results are very sensitive to the Ag content of the layer. The reflectance at high frequency increases rapidly with increasing Ag content of the coating layer. The results are also sensitive to the effectivemedium formulae used to model the coating layer. Good agreement is obtained with the Bruggeman formula, but no reasonable agreement is obtained if we use the Maxwell-Garnett formula instead. The implication is that the silver and the dielectrics in the coating probably form interpenetrating connected networks (appropriate to the microgeometry implied by the Bruggeman formula), rather than nanospheres of one kind embedded in a host of the other (appropriate to the microstructure implied by the Maxwell-Garnett formula).

Features in the measured spectrum can be related to the underlying structure. The photonic band structure, calculated with no absorption, is shown in figure 6. The FCC [111] direction corresponds to the $\Gamma \mathrm{L}$ direction in the band structure, where we find a directional gap ranging from 730 to $1160 \mathrm{~cm}^{-1}$. We thus expect strong reflection in this range. The measured reflectance spectrum does indeed show a high reflectance in this frequency region, in agreement with the position of the spectral gap. Also from the band structure we expect an absolute gap at about $1500 \mathrm{~cm}^{-1}$. The spectral gap along [111] should extend from 1300 to $1640 \mathrm{~cm}^{-1}$.

2 We have also tried the Maxwell-Garnet formula, but failed to reach the same level of agreement within reasonable ranges of parameters. We believe that the Bruggeman formula gives a better approximation because the Ag network and the air holes probably both form the percolated structure. 


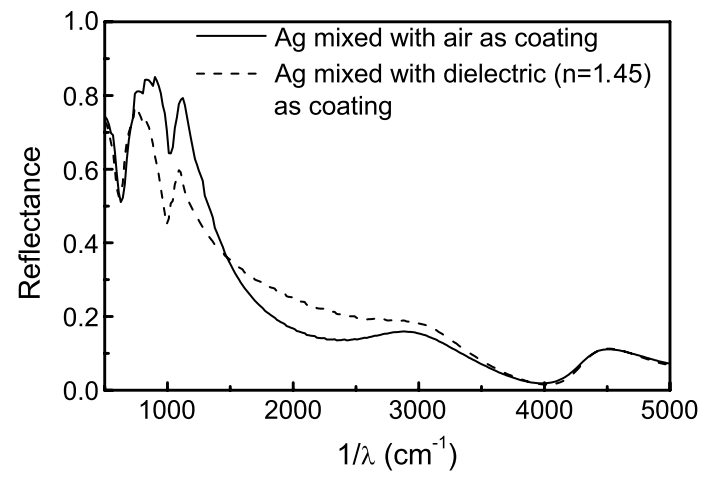

Figure 5. Calculated reflectance of a three-layer slab of coated spheres with the Ag coating layer modelled as an Ag-dielectric mixture, with the dielectric taken as $n=1$ (solid curve) and $n=1.45$ (dashed curve).

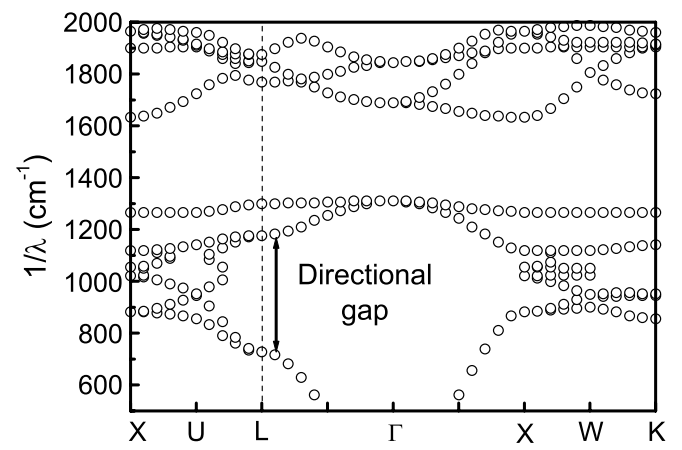

Figure 6. Photonic band structure of an FCC opal determined with the coated micropsheres.

We do not see the signature of the absolute gap in the measured spectrum because of the large absorption of the coated particles at those high frequencies. This can be seen in figure 7 , where we show the calculated absorption cross-section of one coated sphere (glass core with coating) normalized to its cross-sectional area. The absorption is relatively small below $900 \mathrm{~cm}^{-1}$, and thus the strong reflectance due to the directional gap along [111] can be observed. There is a sharp absorption peak at $1010 \mathrm{~cm}^{-1}$ in the calculated absorption, which can be traced to the resonance at the same frequency for the $\mathrm{SiO}_{2}$ core. This sharp absorption feature of an individual sphere manifests itself as a dip at the same frequency (in the high-reflectance regime within the directional gap) in the reflectance spectrum. The absorption cross-section of an individual sphere rises rapidly starting at $1000 \mathrm{~cm}^{-1}$ and peaking at $\sim 1500 \mathrm{~cm}^{-1}$. The strong absorption in this range smears out the absolute gap. The large absorption cross-section is mainly due to the porous nature of the Ag coating layer. If the $\mathrm{Ag}$ coating is uniform, the calculated absorption efficiency is lower than 0.02 within the same frequency range. In that case, the absolute gap should be easily observable. A continuous layer of silver has small absorption because light cannot penetrate into the silver. However, when mixed with a dielectric, the real part of the effective dielectric constant of the composite becomes less negative. Photons can thus penetrate the film and be absorbed.

In order to simulate the experimental spectrum, we use the multiple-scattering approach to calculate the transmission/reflection coefficient. The results are shown in figure 4 , and compare rather well with the measured data. We find that in the higher frequency regime 


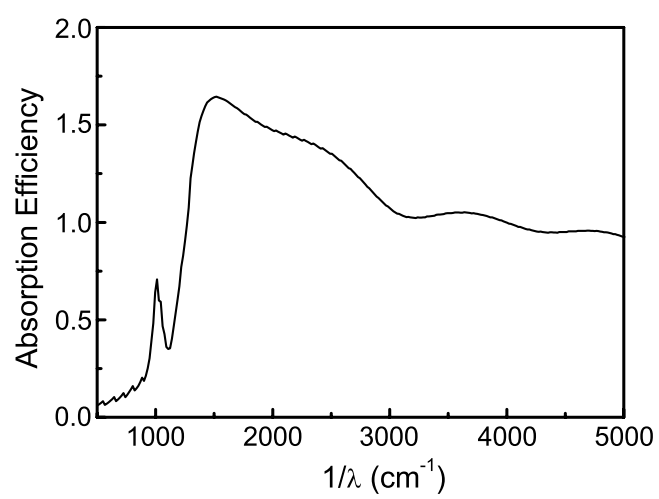

Figure 7. Absorption cross-section of an individual coated sphere normalized to geometric crosssection.

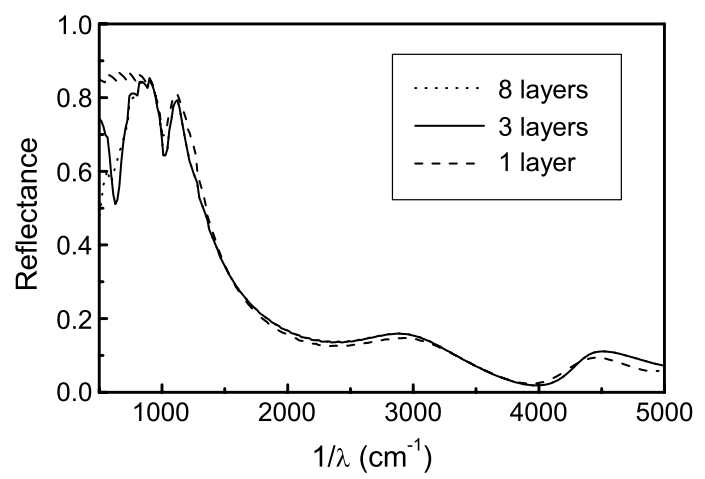

Figure 8. Calculated reflectance spectrum versus the number of stacking layers.

(above $1200 \mathrm{~cm}^{-1}$ ), the large absorption of the particles dominates the reflection spectrum. At frequencies higher than the directional gap, the absorption of the coated particles is so high that the reflection coefficient always remains low even in the frequency region of the absolute gap. In figure 8 , we plot the calculated reflection spectrum versus the number of stacking layers. The reflection spectrum is found to be mainly dominated by the first layer. However, in the high-frequency regime the rapid convergence of the reflectance spectrum with respect to layer thickness does not imply that multiple-scattering effects can be ignored. The multiple scattering within the same layer does contribute to the features in the reflection spectrum, leading to ripples in the high-frequency region. The broad peaks at 3000 and $4500 \mathrm{~cm}^{-1}$ are consequences of the layer structure. Those features can also be found in the measured spectrum. If we were to change the structure of the layer so that it corresponds to an exposed [100] facet instead of the [111] facet, these peaks would shift in frequency.

In figure 9, we compare the calculated reflectance along the normal of the surface plane of three layers of $2 \mu \mathrm{m}$ coated spheres arranged in an FCC[111] structure with the calculated reflectance of a three-layer simple-cubic [100] structure made with exactly the same kind of coated spheres and a lattice constant of $2.4 \mu \mathrm{m}$. We see that there is a rapid drop in reflectance for both cases starting from about $1200 \mathrm{~cm}^{-1}$, which is due to the strong absorption of the coating of a single sphere. The dip at about $1010 \mathrm{~cm}^{-1}$, which is due to the absorption of core of the single sphere, also also appears for both cases. However, the effect of the structural arrangement of the spheres (band structure effects) still plays a role in determining the details of 


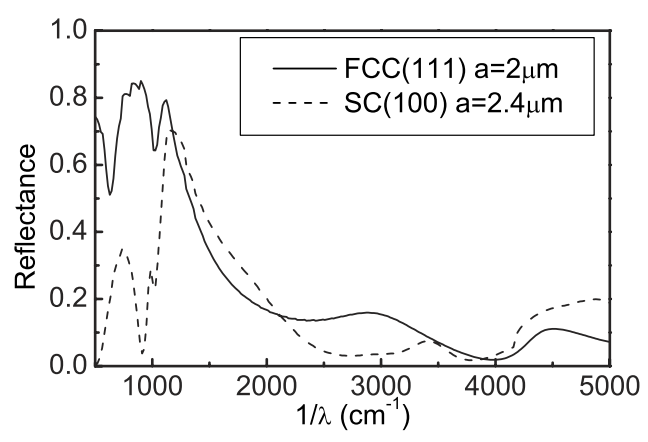

Figure 9. Calculated reflectance spectrum for the same kind of coated spheres arranged in different structures and orientations. See text for details.

the reflection spectra. We see that even in the high absorption region of higher frequencies, the two different structures give different ripple structures. At lower frequencies, the absorption is small and the high reflectance reflects the position of the directional band gaps of the structural arrangement of the spheres. The changes in the simple-cubic reflectance spectra can be traced to an upward shift of the direction gap in the simple-cubic structure.

\section{Discussion}

Opal slabs consisting of coated microspheres packed in an FCC structure have been fabricated. Their optical properties were measured in the IR frequency regime. Comparisons with theoretical calculations show that the Ag coating layer is best modelled by an Ag-dielectric composite. The directional gap expected of a metal-coated FCC opal system is manifested as a high-reflectance region in the spectrum, but the high-frequency absolute gap is smeared out by absorption, due to the porous nature of the Ag coating. If a higher Ag content can be achieved in the coating layer, the absolute gap should be easily observable.

We note that disorder should not be a major factor in the observed low reflectance in the high-frequency region, since the spectrum is measured on a selected, highly ordered single domain as judged by optical microscope inspection.

The key issue to realize the absolute gap is to improve the quality of the coating layer, such as increasing the metal content and the homogeneity. For example, a layer of higher homogeneity may be facilitated by adding surfactants to enhance the efficiency of the coating process. Also, other metals, e.g. gold or copper, can be used as the coating because of their well-developed technology, even though they may not be as reflective as silver at optical frequencies. Last but not least, the fact that the metallo-dielectric crystal, as prepared, may have high absorption in the absolute gap region but high reflectivity in the directional gap region may be useful for some other purposes. We know from Kirchhoff's law that a good absorber is also a good emitter. That means that the metallo-dielectric crystals can, when heated to a certain temperature, emit more photons at higher frequencies than at longer IR frequencies when compared with a black-body radiator. Such modification of thermal emission spectra may be useful on some occasions.

\section{Acknowledgments}

We acknowledge the financial support of RGC Hong Kong grants HKUST6122/98P, HKUST6145/00P and N_HKUST033/00. Technical help from N Wang is also acknowledged. 


\section{References}

[1] See, for example,

Soukoulis C M 1993 Photonic Band Gaps and Localization (New York: Plenum)

Soukoulis C M 1993 J. Opt. Soc. Am. B 10 208-408

Soukoulis C M 1996 Photonic Band Gap Materials (Dordrecht: Kluwer)

Yablonovitch E 1987 Phys. Rev. Lett. 582059

John S 1987 Phys. Rev. Lett. 582486

Joannopoulos J D, Meade R D and Winn J 1995 Photonic Crystals (Princeton, NJ: Princeton University Press)

[2] See, for example,

Cheng C C et al 1996 Phys. Scr. T 6817

Noda S and Sasaki A 1997 Japan. J. Appl. Phys. 361907

Lin S Y et al 1998 Nature 394251

[3] For a review, see, for example,

van Blaaderen A 1998 Science 282887 and references therein

Wijnhoven J E G and Vos W L 1998 Science 281802

Imhof A and Pine D J 1997 Nature 389948

Velev A et al 1997 Nature $\mathbf{3 8 9} 447$

Zakhidov A et al 1998 Science 282897

Holland B T, Blanford C T and Stein A 1998 Science 281538

van Blaaderen A 1998 Science 282887

Subramania G et al 1999 Appl. Phys. Lett. 743933

[4] See, for example,

Smith D R et al 1994 Appl. Phys. Lett. 65645

McIntosh K A et al 1997 Appl. Phys. Lett. 702937

McCalmont J S et al 1996 Appl. Phys. Lett. 682759

Sievenpiper D F, Sickmiller M E and Yablonovitch E 1996 Phys. Rev. Lett. 762480

Sievenpiper D F, Yablonovitch E, Winn J N, Fan S, Villeneuve P R and Joannopoulos J D 1998 Phys. Rev. Lett. 802829

Moroz A 2000 Europhys. Lett. 50466

Graf C and van Blaaderen A 2002 Langmuir 18524

Garcia-Santamaria F et al 2002 Langmuir 184519

Liang Z, Susha A S and Caruso F 2002 Adv. Mater. 141160

Moroz A 2002 Phys. Rev. B 66115109

[5] Zhang W Y, Lei X Y, Wang Z L, Zheng D G, Tam W Y, Chan C T and Sheng P 2000 Phys. Rev. Lett. 842853

[6] Andreeva L P 1990 Sov. J. Opt. Technol. 57 254-6

Sodervall B V and Lundeberg T 1995 US Patent Specification 5395651

[7] Wagner C D, Riggs W M, Davis L E, Moulder J F and Mullenberg G E 1979 Handbook of X-ray Photoelectron Spectroscopy (Eden Prairie, MN 55344: Perkin-Elmer Corporation, Physical Electronics Division)

[8] Park S H and Xia Y 1999 Langmuir 15266

[9] There are many different formulations: see, for example,

Ohtaka K 1980 J. Phys. C: Solid State Phys. 13667

Stefanou N, Karathanos V and Modinos A 1992 J. Physique 47389

Wang X D, Zhang X G, Yu Q L and Harmon B N 1993 Phys. Rev. B 474161

Tayeb G and Maystre D 1997 J. Opt. Soc. Am. A 143323

Li L M and Zhang Z Q 1998 Phys. Rev. B 5815589

[10] Stefanou N, Yannopapas V and Modinos A 1998 Comput. Phys. Commun. 11349

Yannopapas V, Modinos A and Stefanou N 1999 Phys. Rev. B 605359

[11] Bruggeman D A G 1935 Ann. Phys., Lpz. 24636

[12] Palik E D 1985 Handbook of Optical Constants of Solids (New York: Academic)

IR data are taken from: Ordal M A et al 1983 Palik Appl. Opt. 221099 